\title{
Microstructural examination of shear localisation during high strain rate deformation of Alloy 718
}

\author{
Joakim Johansson ${ }^{1}$, Christer Persson ${ }^{1}$, Haiping Lai ${ }^{2}$, Magnus Hörnqvist Colliander ${ }^{2,3, *}$ \\ ${ }^{1}$ Materials and Manufacturing Technology, Chalmers University of Technology, SE-412 96 \\ Gothenburg, Sweden \\ ${ }^{2}$ Applied Physics, Chalmers University of Technology, SE-412 96 Gothenburg, Sweden \\ ${ }^{3}$ GKN Aerospace Engine Systems, SE-461 81, Trollhättan, Sweden \\ *Corresponding author. \\ E-mail address: magnus.colliander@chalmers.se (M. Colliander)
}

\begin{abstract}
Chip formation during metal cutting involves high strain rates and large deformations. Under many conditions, the deformation is concentrated in narrow bands due to shear localisation from adiabatic heating. In order to understand the localisation process, it is necessary to increase the knowledge regarding the microstructural evolution during deformation. However, the deformation that occurs during chip formation is hard to measure. Therefore, this study utilises top-hat specimens deformed at high strain rates in order to generate localised shear bands in the Ni-based superalloy Alloy 718, with defined measurable deformation. The resulting shear bands in the top-hat specimens are compared with those generated in metal cutting chips and studied in order to characterise the deformation occurring at a microstructural level. The resulting microstructures in the top-hat specimens and machining chips are found to be similar, with heavily localised deformation into narrow shear bands and homogeneously sheared microstructure adjacent to the bands. The centres of the shear bands are heavily deformed with ultra-fine grains, indicating dynamic recrystallisation during the deformation. The results indicate that the shear deformation produced by high strain rate testing of top-hat specimens can provide an excellent means of replicating the conditions for shear localisation during metal cutting. However, care should be taken to design the tests so that the local conditions are representative in terms of strains and strain rates.
\end{abstract}

Keywords: Nickel-based superalloy, Adiabatic shear bands, Dynamic recrystallisation, Metal cutting, Electron microscopy, Split-Hopkinson pressure bar 


\section{Introduction}

Metal cutting is one of the most value adding operation processes for producing high quality products and involves removing chips with a hard insert to get the desired shape of the product. During metal cutting, the machining conditions influence the cut chip morphology. The chip morphology in turn influences for example the cutting forces, tool wear, surface characteristics and chip breakage and removal $[1,2]$. At low cutting speeds the chips often show a continuous morphology, which changes to segmented chips at increased cutting speed [3]. Chip segmentation is caused by localised instabilities, where the majority of the deformation is confined to the thin bands, with wider segments of less deformed material in between. The localised shear bands are the preferential locations for crack initiation and propagation, and thereby contribute to chip breakage.

The traditional explanation behind the localisation of the material flow is connected to the short duration of the deformation, where the generated heat during plastic work has little time to dissipate to surrounding material. If the temperature build-up is sufficiently high, the thermal softening can overcome the strain and strain-rate hardening effects, leading to locally reduced material strength and continued strain localisation. The bands are often referred to as adiabatic shear bands due to the apparent adiabatic heating in the shear zone [4]. The tendency for shear localisation increases at high strain rates for materials with high strength, where the deformation produces considerable heat.

Superalloys, which maintain their high strength levels at elevated temperatures, represent a class of materials that are difficult to machine. During machining, superalloys produce considerable heat, which in combination with poor thermal conductivity gives a high local temperature that increases the tool wear and therefore limits the material removal rate. The chip formation process is complex and the deformation that occurs is hard to determine accurately. However, the deformation occurring in the primary shear zone of the cut chips is reported to occur at a strain rate in the order of $10^{3}$ to $10^{6} \mathrm{~s}^{-1}$ during typical machining conditions and experiences strain in the order of 1 to 10 [5]. The maximum temperature is reported to be as high as between 800 and $1000^{\circ} \mathrm{C}[6]$.

For these reasons, superalloys are prone to localised shear band formation during high-speed machining [3]. Most of the studies of shear localisation during machining of superalloys have concentrated on experimental investigations of the formation of segmented chips [7,8], and phenomenological modelling of the localisation process, e.g. [9]. Other aspects of shear localisation have been studied under ballistic conditions, both experimentally and numerically, in order to study containment issues [10].

However, so far little effort has been devoted to the detailed microstructural evolution in the bands, which is necessary in order to understand the mechanisms of formation. Since the local deformation situation during machining is complex with several interacting forces and processes, it is virtually impossible to experimentally determine the local conditions. As a means to understand the local deformation processes, other test methods can be used to create shear bands with a better-controlled thermo-mechanical history. This requires the transferability between two situations to be verified. The purpose of the present study is to compare the 
microstructures in and around localised shear bands in Alloy 718 generated by Split-Hopkinson testing with those observed after machining.

In this study, top-hat samples are subjected to dynamic deformation in a Split-Hopkinson test equipment. The geometry of the samples promotes shear localisation and results in deformation focused in a narrow shear band (approximately $4-5 \mu \mathrm{m}$ wide). The shear bands produced are very similar to the bands observed in chips from metal cutting trials, and the strain rate in the localised bands are similar for both cases at around $10^{6} \mathrm{~s}^{-1}$. The deformed microstructures in the top-hat sample and cut chip show similar deformation structures where the microstructure around the localised band exhibits a shear texture, with very sharp transition between the shear band and the adjacent material. Inside the shear bands, dynamically recrystallised grains in the order of 20-300 $\mathrm{nm}$ in diameter are observed, which exhibit a clear shear texture. The average size of the recrystallised grains appears to be slightly smaller in the chips.

This study indicates that the microstructure created during Split-Hopkinson top-hat tests and metal cutting trials of Alloy 718 are similar, in terms of both macroscopic shear band appearance and local deformation structure and texture within the bands. Provided that the tests are designed to mimic the local deformation conditions during machining sufficiently well, high strain rate tests using the top-hat geometry could potentially be used to produce localised shear bands with a more well-controlled history in order to study the localisation process.

\section{Material}

The material used in this work is Alloy 718, a precipitation hardened iron nickel-based superalloy commonly used in aero and land-based gas turbines [11, 12]. The material was obtained in the form of wrought bars, conforming to AMS 5662M [13]. A hot rolled $15.88 \mathrm{~mm}$ bar was used to produce the top-hat samples and a wrought $126 \mathrm{~mm}$ bar was used for the metal cutting experiments. The grain sizes were measured to ASTM grain size 11 and 9, respectively, for the small and large diameter bars [14]. The microstructures and textures of the two bars are shown in Figure 1. Whereas the large bar showed a uniform texture, the small bar had a noticeable but weak $<011>\|$ RD texture. The chemical compositions of the two bars are shown in Table I [15].

The material was solution heat treated at $954{ }^{\circ} \mathrm{C}$ and water quenched. For the small bar, a soaking time of 1 hour was used, whereas the thick bar was solutionised for 2 hours to provide extra time for heating of the large mass. The solution treatment was followed by a precipitation treatment at $718^{\circ} \mathrm{C}$ for 8 hours followed by furnace cooling to $621^{\circ} \mathrm{C}$, and subsequently held for a total heat treatment time of 18 hours and then air cooled, in accordance to material standard [13]. 


\section{Experimental}

\subsection{Testing procedures}

The top-hat samples were tested in a Split Hopkinson test rig in compression. The Split Hopkinson rig consists of two long elastic bars, the incident and transmitter bars, and one shorter striker bar. The striker bar is fired at the incident bar and initiates an elastic wave, where the wave travels along the incident bar and eventually hits the sample. Part of the wave is transferred through the sample to the transmitter bar and part is reflected. By measuring the strains with strain gauges on the incident and transmitter bars, we can calculate the force and strain rate of the sample. Figure 2 show a schematic of the test setup, and the technique is extensively described in literature $[16,17]$. In order to ensure that the sample studied has only been deformed by only one stress pulse, the setup utilises a traditional lead brick that makes the transmitter bar bounce-back sluggish and a stress reversal technique removing the incident bar after initial pulse as described by Nemat-Nasser et al. [17].

The samples used in this study are chosen in order to generate controlled shear bands, where the geometry forces the majority of the deformation to occur in shear in a limited region. The geometry of the samples used is seen in Figure 3. During testing, the smaller diameter top is forced in the cut pocket at the bottom, concentrating the deformation to a narrow region enabling heavy shear localisation that, in combination with high strain rate, produces shear bands. To limit the deformation in the shear band, a stopper ring is placed around the top, thus limiting the amount it can be pushed into the cut pocket to $1 \mathrm{~mm}$.

The metal cutting experiment was performed in a SOMAB Optimab $400 \mathrm{CNC}$ turning lathe. Greenleaf Alumina-SiC whiskers reinforced inserts were used, and a cutting speed of 240 $\mathrm{m} / \mathrm{min}$, feed of $0.1 \mathrm{~mm} / \mathrm{rev}$ and a depth of cut of $2 \mathrm{~mm}$ in orthogonal dry cutting. The cutting was done on $2 \mathrm{~mm}$ wide flanges produced with a separate grooving insert [18]. The combination of machining parameters was chosen to produce a serrated chip morphology [18].

\subsection{Microstructural characterisation}

The samples were sectioned, ground and polished to a mirror finish with a final step using colloidal silica suspension to get a slight etching effect to reveal the shear band and microstructure during initial optical microscopy. The silica suspension polish also removes surface deformation induced by previous grinding and polishing steps, enabling electron backscatter diffraction (EBSD) analysis in a scanning electron microscope (SEM). To allow further observation of the microstructure and shear bands, electrochemical etching in oxalic acid was used [19], where the shear band appears as a white etching band and the delta phase is revealed.

Optical microscopy was performed in a Leica Leitz DMRX conventional optical microscope and low magnification overview images were taken in a Zeiss SteREO Discovery V20 stereooptical microscope. Both are equipped with digital cameras AxioCam MRc 5 from Carl Zeiss.

The SEM used for microstructural observations was an LEO 1550 Gemini field emission gun SEM (FEG-SEM) equipped with EBSD detector from Oxford Instruments. EBSD in the SEM 
was used to map the area adjacent to the shear localisation. To obtain texture information with higher spatial resolution than achievable though conventional EBSD, transmission Kikuchi diffraction (TKD) in the SEM was utilised. Since TKD is performed on thin foils in transmission mode, spatial resolution down to 2-5 nm can be achieved [20, 21]. TKD was performed using an HKL Channel 5 EBSD system on a Leo Ultra 55 FEG-SEM operated in high-current mode at $30 \mathrm{kV}$ with large aperture $(120 \mu \mathrm{m})$. A special sample holder was used to hold the thin foils at a $20^{\circ}$ angle to allow the transmitted Kikuchi patterns to be collected by the Nordlys II EBSD detector. A step size of $15 \mathrm{~nm}$ was used in order to optimise the resolution without increasing the total acquisition time too much, as high resolution renders a high sensitivity to drift. The band contrast from the recorded Kikuchi patterns can be used to image the structure of the material, since it is sensitive to the presence of high and low angle grain boundaries. To image the structure in a higher resolution, transmission electron microscopy (TEM) was performed in an FEI Titan 80-300 instrument operated at $300 \mathrm{kV}$.

Specimens for TKD and TEM were produced by lift-out in a FEI Versa3D Lovac dual-beam focused ion beam/scanning electron microscope (FIB/SEM) instrument. Thin foils, which contained the region of interest, were extracted by FIB milling, attached to a $\mathrm{Cu}$ TEM grid, and thinned down to electron transparency though ion milling. The same foils were used for TEM and TKD investigations.

\section{Results}

\subsection{Top-hat tests}

The deformed specimen in Fig. 4 shows strong localisation to the shear regions. The shear band becomes clearly visible by etching, and the width was measured to roughly 4 to $5 \mu \mathrm{m}$ on average. Although some elongation and rotation of the grains closest to the shear band can be seen, it is difficult to discern any clear transition zone between the surrounding material and the band. In the global force displacement curve, Fig. 5, the point of localisation is indicated by the drop in load and strain rate at a displacement of around $0.3 \mathrm{~mm}$. It is unlikely that the subsequent increase in load is due to strain hardening since localisation depends on material softening in the band. The load increase could be a result of the increasing compressive load when the top is forced into the ring.

Before the occurrence of strain localisation, the shear deformation is distributed over a larger volume, usually denoted the shear affected zone (SAZ) [22]. Figure 6(a) shows an EBSD map of the SAZ surrounding the shear band, exhibiting a pronounced shear texture throughout. The shear plane normal (SPN) and shear direction (SD) are indicated in the pole figures. The shear band is clearly seen as the black region (un-indexed pixels) in the centre of the map. As in the optical micrograph, no distinct transition zone can be seen. A close-up of the region adjacent to the band further confirms this (Fig. 7(a)). Typically, a rather wide zone with increasing rotation and elongation of the grains is observed [23], but Fig. 7(a) shows a rather abrupt transition from relatively homogeneous shear. It should be noted that the small grains at the very boundary between the surrounding and the un-indexed band are a result of the data processing and cleanup, and should not be considered in the interpretation. The abrupt transition is also clearly seen 
in the band contrast map of the same region (Fig. 8). The shear texture is preserved in the region closest to the band (Fig. 7(b)).

The structure within the shear band could not be resolved in the SEM. The band contrast map (Fig. 8) seems to indicate the presence of very fine grains, but they cannot be further resolved. To clarify the internal structure of the shear band, thin foils were extracted by FIB milling, with the foil plane lying in the band, and investigated by TKD. Figure 9(a) shows the raw (unprocessed) EBSD map from such a foil, and the corresponding pole figures show a clear shear texture (Fig. 9(b)). Since the number of un-indexed pixels was quite high, the unprocessed data was used for calculation of the texture in order not to introduce artefacts from noise reduction and clean-up. The band contrast map, shown in Fig. 9(c), can nonetheless be used to image feature of the substructure, which consists of ultra-fine equiaxed grains with a size in the order of $50-300 \mathrm{~nm}$.

Further details are revealed by the TEM bright-field micrographs. Figure 10(a) shows the boundary between the shear band and the adjacent material, with no obvious transition zone. Figure 10(b) and (c) shows an overview and close-up, respectively, of the ultra-fine grains within the band. The grains are confirmed to be equiaxed, with sizes down to around $20 \mathrm{~nm}$.

\subsection{Machining chips}

The chip from the machining trials (Fig. 11) shows a clear segmentation where the shear band divides segments with less deformed material. The appearance on a microstructural level is very similar to the deformed top-hat sample, with a very narrow shear band with similar width for the two cases.

The area between the shear bands shows a distinct shear texture from the homogeneous deformation, as seen in Fig. 12, and, as in the case of the top-hat specimens, no pronounced transition zone can be seen. The shear texture has been seen previously in metal cutting chips [24]. A close-up of the regions closest to the shear band (Fig. 13) shows grains in the process of subdivision by shearing of different regions delineated by low angle grain boundaries (arrows). As before, the small grains at the very boundary between the surrounding and the unindexed band are a result of the data processing and clean-up, and should not be considered in the interpretation.

TKD investigations of thin foils from within the band show a shear texture based on the pole figures from the raw data, see Fig. 14. Ultra-fine equiaxed grains can be seen from the band contrast map. The grains in the chip appear to be slightly smaller than in the top-hat specimens, most of them being around 50-200 $\mathrm{nm}$. The TEM investigation also confirms the similarities in deformation structure between the chip and the top-hat specimen (Fig. 15). There is no obvious transition zone between the band and the surroundings, and the structure is equiaxed with grains as small as $20 \mathrm{~nm}$.

\section{Discussion}

The deformed microstructure formed in the top-hat sample and chip from metal cutting shows similar features. The formed microstructure shows a shear texture in the bands and adjacent 
material. The shear bands show grain refinement with grains as small as $20 \mathrm{~nm}$, although the majority of the grains in the shear bands are in the range of 50-300 $\mathrm{nm}$. On average, the recrystallised grain size appears to be slightly smaller in the machining chip. This is presumably due to differences in deformation history, as it is likely that both the deformation and temperature history can influence the grain size of the shear bands.

In order to ensure the usefulness of studying top-hat samples to get a better understanding of shear localised processes in metal cutting, it is necessary to produce a deformation history similar to that which occurs during the machining operations. It isn't possible to measure the deformation that occurs in the shear bands, and the strain rate and strain in the shear bands cannot be determined with accuracy as the deformation within the band are unlikely to be uniform [25]. This, in combination with the fact that the duration under which the shear band is formed is hard to determine as the shear band is preceded by some amount of uniform deformation before the shear band is initiated, makes any calculation only approximate. In this study, the strain rate and strain are calculated using a model used, among others, by Yan et al. [26] and Andrade et al. [27] where the shear strain is taken as the displacement of the shear band divided by the width of the band, and the strain rate is the strain divided by the duration of the deformation. With this approximation, the strain rate in the chip and top-hat sample are similar and in the order of $10^{6} \mathrm{~s}^{-1}$, and the shear strains, $\gamma$, of approximately 16 for the chip and 120 for the top-hat sample. The true strain, $\varepsilon$, can be calculated as [27]

$$
\varepsilon=\ln \sqrt{1+\gamma+\frac{\gamma^{2}}{2}}
$$

giving strains of 2.5 and 4.4 for the chip and top-hat, respectively. The difference in strain is a result of the larger displacement of the shear band in the top-hat sample. No large differences are seen in the studied microstructures, except the tendency for the recrystallised grain size in the cut chip to be slightly smaller. The similarities in the two microstructures indicate that the differences in strain at these levels are likely to have little effect on the deformed microstructure. By using numerical simulations, it would be possible to have a better estimate of the local deformations in the sample and therefore possible to optimise the specimen geometry and test conditions in order to better fit the metal cutting cases in term of the deformation and temperature history. By performing this further optimisation of future tests, we can ensure that the compared microstructures are from a similar deformation history.

There is a large spread in the reported widths and shear band appearance in literature, where the width reported stretches from more than $100 \mu \mathrm{m}$ for e.g. Ti-bearing IF-steel [23] to the 4-5 $\mu \mathrm{m}$ seen in the present work. The transition zone to the shear band also varies in literature, where some find a clear transition with gradually refined structure and in our study where there is a diffuse and very narrow transition zone [23]. All this indicates that the shear localisation behaviour is highly material dependent and possibly also process dependent, and needs further research in order to be explained. The recrystallisation seen in the shear zone is seen in a variety of materials subjected to dynamic deformation, e.g. [23, 28, 29]. The shear texture in the recrystallised structure suggests that a rotational form of dynamic recrystallisation (DRX) is 
active, where the grains are heavily stretched, subdivided and form new equiaxed grains, as suggested by Hines et al. [30].

The initiation of shear bands and the resulting drop in load carrying capability is of importance in modelling. By gaining a better understanding of the deformation behaviour in the shear band, and the mechanisms that initiate the localisation, there is a possibility to better simulate and predict the shear localisation behaviour. There are a few different theories behind shear localised bands where the traditional explanation is based on local adiabatic heating in which the thermal softening overcomes the strain hardening and therefore promotes further deformation in that narrow band [4]. However, it is debatable whether the local adiabatic heating alone is able to account for the localisation and recrystallisation. A study by Hines and Vecchio [31] observed localisation and recrystallisation for top-hat copper samples tested at 77 $\mathrm{K}$ that did not reach more than $340 \mathrm{~K}$ during testing, which is generally considered too low for thermally activated recrystallisation. Another interesting possible mechanism for shear band initiation is proposed by Rittel [32], claiming that DRX actually precedes thermal softening and is the precursor for shear band localisation. He makes this claim with studies of Ti6Al4V among other alloys, where DRX can be observed prior to the formation of shear bands [33].Yet other studies point to the possibility that the shear band is initiated by cracks forming at the free surface, with observations showing that the shear band often originates from the surface and grows inwards [7, 8]. It is possible that the mechanism and shear band formation behaviour is material, deformation and temperature sensitive. Dynamic testing using top-hat samples is useful for further studies as the geometry and deformation history is more controllable than in typical machining trials, and can therefore be tailored to investigate the different stages of shear band localisation.

\section{Conclusions}

The microstructures resulting from metal cutting and dynamic testing of top-hat specimens both show very narrow 4-5 $\mu \mathrm{m}$ wide shear localised bands with recrystallised grains down to the order of 20-300 $\mu \mathrm{m}$ and a very narrow transition zone to adjacent material. Grains as small as $20 \mathrm{~nm}$ can be observed in both specimens, but the average recrystallised grain size appears to be slightly smaller in the chips. The shear texture in the bands indicates that the recrystallisation has occurred through a rotational mechanism.

The strain and strain rate was similar in the top-hat sample and cut chip, with strain rates in the order of $10^{6} \mathrm{~s}^{-1}$, and true strains of 4.4 and 2.5, respectively, in the shear band. Although the chip shows a lower level of strain in the shear band, the deformation is believed to be comparable as the deformed microstructures have a similar appearance.

This study show that it is possible to produce shear localised deformation in measurable, specialised test equipment that shows similar deformation appearance, grain size and texture as that produced in metal cutting chips. This further facilitates detailed studies of underlying phenomena, e.g. initiation and propagation of shear bands. 


\section{Acknowledgement}

Split-Hopkinson testing of the top-hat sample was performed at the University of California San Diego with the help of Professor Kenneth Vecchio. The metal cutting experiments were performed by Albin Hagberg and Peter Malm as part of their M.Sc. thesis under the supervision of Stefan Cedergren at Chalmers University of Technology.

The project has been financially supported by Family Ekman's Research Donation, which is gratefully acknowledged. The testing has been financially supported by VINNOVA and GKN Aerospace in collaboration in the NFFP5 program. 


\section{References}

1. Dong, G., et al., Study of cutting deformation in machining nickel-based alloy Inconel 718. International Journal of Machine Tools and Manufacture, 2011. 51(6): p. 520527.

2. $\quad \mathrm{Su}, \mathrm{G}$., et al., Influences of chip serration on micro-topography of machined surface in high-speed cutting. International Journal of Machine Tools and Manufacture, 2015. 89(0): p. 202-207.

3. Komanduri, R. and T.A. Schroeder, On Shear Instability in Machining a Nickel-Iron Base Superalloy. J. Manuf. Sci. Eng, 1986. 108: p. 93-100.

4. Wright, T.W., The physics and mathematics of adiabatic shear bands 2002, Cambridge, UK Cambridge University Press.

5. Guo, Y.B., An integral method to determine the mechanical behavior of materials in metal cutting. Journal of Materials Processing Technology, 2003. 142(1): p. 72-81.

6. Jafarian, F., et al., Finite element simulation of machining Inconel 718 alloy including microstructure changes. International Journal of Mechanical Sciences, 2014. 88(0): p. 110-121.

7. Vyas, A. and M.C. Shaw, Mechanics of Saw-Tooth Chip Formation in Metal Cutting. Journal of Manufacturing Science and Engineering, 1999. 121(2): p. 163-172.

8. Nakayama, K., FORMATION OF "SAW-TOOTHED CHIP" IN METAL CUTTING. 1974: p. 572-577.

9. Chung, D.T., S. Nemat-Nasser, and L.M. Taylor, Phenomenological Modelling of Rate-Dependent Plasticity for High Strain Rate Problems, in Computational Mechanics '88, S.N. Atluri and G. Yagawa, Editors. 1988, Springer Berlin Heidelberg. p. 279-282.

10. Pereira, J.M. and B.A. Lerch, Effects of heat treatment on the ballistic impact properties of Inconel 718 for jet engine fan containment applications. International Journal of Impact Engineering, 2001. 25(8): p. 715-733.

11. Baker, J.F., The Initial Years of Alloy 718 - A GE Perspective, in Superalloy 718 Metallurgy and Application, TMS 1989.

12. Loria, E., Recent developments in the progress of superalloy 718. JOM, 1992. 44(6): p. 33-36.

13. AMS 5662M, Aerospace material specification. 2009, SAE Aerospace.

14. Cedergren, S., et al., The effects of grain size and feed rate on notch wear and burr formation in wrought Alloy 718. The International Journal of Advanced Manufacturing Technology, 2013. 67(5-8): p. 1501-1507.

15. Cedergren, S., et al., The effects of grain size and feed rate on notch wear and burr formation in wrought Alloy 718. The International Journal of Advanced Manufacturing Technology, 2012: p. 1-7.

16. Gray, G.T., Classic Split-Hopkinson Pressure Bar Testing in ASM Handbook, Volume 08 - Mechanical Testing and Evaluation, H. Kuhn and D. Medlin, Editors. 2000, ASM International. p. 462-476.

17. Nemat-Nasser, S., B.I. Jon, and J.E. Starrett, Hopkinson Techniques for Dynamic Recovery Experiments. Proceedings: Mathematical and Physical Sciences, 1991. 435(1894): p. 371-391.

18. Hagberg, A. and P. Malm, Material Deformation Mechanisms during Machining of Superalloys, in Department of Materials and Manufacturing Technology. 2010, CHALMERS UNIVERSITY OF TECHNOLOGY.

19. Small, K.B., D.A. Englehart, and T.A. Christman, Guide to etching specialty alloys. Advanced Materials \& Processes, 2008. Vol. 166 (Issue 2). 
20. Trimby, P.W., Orientation mapping of nanostructured materials using transmission Kikuchi diffraction in the scanning electron microscope. Ultramicroscopy, 2012. 120(0): p. 16-24.

21. Keller, R.R. and R.H. Geiss, Transmission EBSD from $10 \mathrm{~nm}$ domains in a scanning electron microscope. Journal of Microscopy, 2012. 245(3): p. 245-251.

22. Cerreta, E.K., et al., Microstructural examination of quasi-static and dynamic shear in high-purity iron. International Journal of Plasticity, 2013. 40: p. 23-38.

23. Lins, J.F.C., et al., An EBSD Investigation on Deformation-Induced Shear Bands In Ti-Bearing IF-Steel Under Controlled Dynamic-Loading Conditions. Materials Science Forum, 2005. Vols. 495-497: p. pp. 393-398.

24. Basu, S. and M. Ravi Shankar, Crystallographic Textures Resulting from Severe Shear Deformation in Machining. Metallurgical and Materials Transactions A, 2015. 46(2): p. 801-812.

25. Chen, S., et al., Prediction of the initial thickness of shear band localization based on a reduced strain gradient theory. International Journal of Solids and Structures, 2011. 48(21): p. 3099-3111.

26. Yan, D.P., et al., On Quantifying the Strain Rate During Chip Formation When Machining Aerospace Alloy Ti-5553. Procedia CIRP, 2013. 8(0): p. 123-128.

27. Andrade, U., et al., Dynamic recrystallization in high-strain, high-strain-rate plastic deformation of copper. Acta Metallurgica et Materialia, 1994. 42(9): p. 3183-3195.

28. Yang, Y., et al., Microstructural characterization and evolution mechanism of adiabatic shear band in a near beta-Ti alloy. Materials Science and Engineering: A, 2011. 528(6): p. 2787-2794.

29. Wang, B.F., et al., An EBSD investigation on deformation-induced shear bands in a low nickel austenitic stainless steel under controlled shock-loading conditions. Materials Science and Engineering: A, 2014. 610(0): p. 301-308.

30. Hines, J., K. Vecchio, and S. Ahzi, A model for microstructure evolution in adiabatic shear bands. Metallurgical and Materials Transactions A, 1998. 29(1): p. 191-203.

31. Hines, J.A. and K.S. Vecchio, Recrystallization kinetics within adiabatic shear bands. Acta Materialia, 1997. 45(2): p. 635-649.

32. Rittel, D., A different viewpoint on adiabatic shear localization. Journal of Physics D: Applied Physics, 2009. 42(21): p. 214009.

33. Rittel, D., P. Landau, and A. Venkert, Dynamic Recrystallization as a Potential Cause for Adiabatic Shear Failure. Physical Review Letters, 2008. 101(16): p. 165501. 
Table I. Chemical composition of studied Alloy 718

\begin{tabular}{llllllllllllll}
\hline \multicolumn{2}{l}{ Top-hat sample } & & & & & & & & & & & \\
\hline Ni & Cr & Co & Fe & C & Mo & Al & Ti & Nb & B & Mn & Si & Cu \\
\hline 52.49 & 18.53 & 0.12 & 18.73 & 0.025 & 3.05 & 0.57 & 0.98 & 5.29 & 0.004 & 0.04 & 0.05 & 0.03 \\
\hline \multicolumn{2}{l}{ Metal cutting chip } & & & & & & & & & & \\
\hline Ni & Cr & Co & Fe & C & Mo & Al & Ti & Nb & B & Mn & Si & Cu \\
\hline Bal & 18.36 & 0.33 & 17.49 & 0.04 & 3.15 & 0.56 & 0.92 & 5.46 & 0.001 & 0.09 & 0.05 & 0.14 \\
\end{tabular}



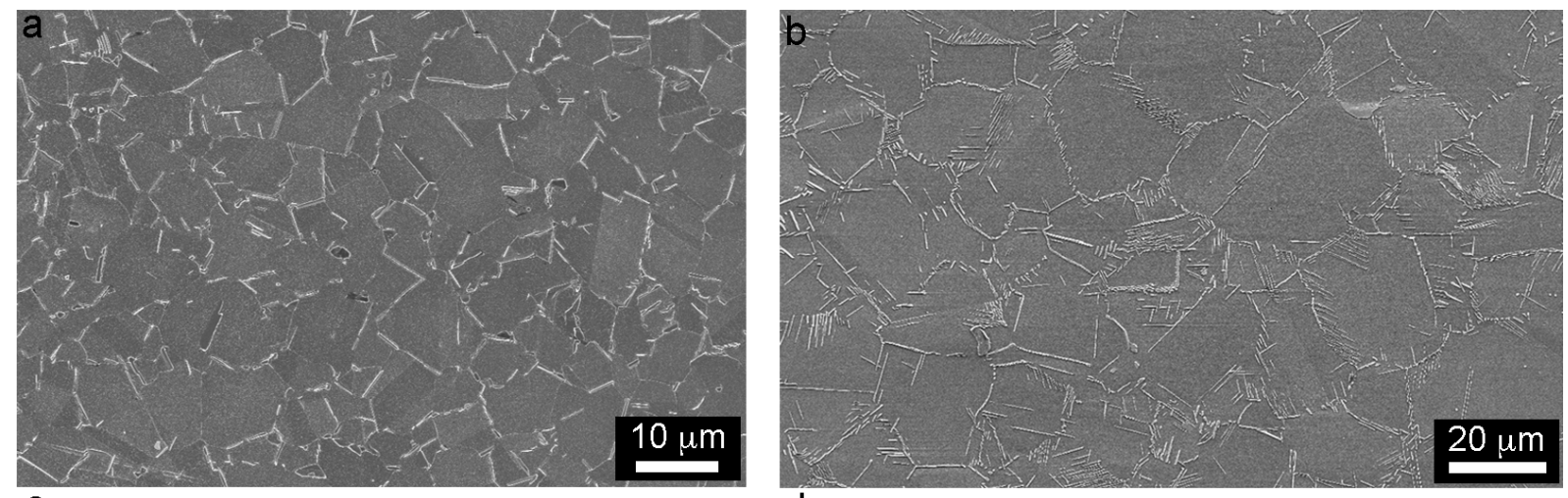

C

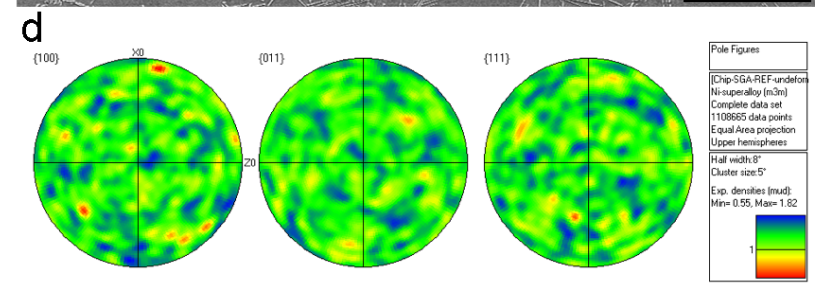

Figure 1. SEM image of un-deformed microstructure of the material used for (a) Split Hopkinson top-hat testing, and (b) machining trials. (c) and (d) show the corresponding pole figures, with the rolling direction normal to the plane of the paper.

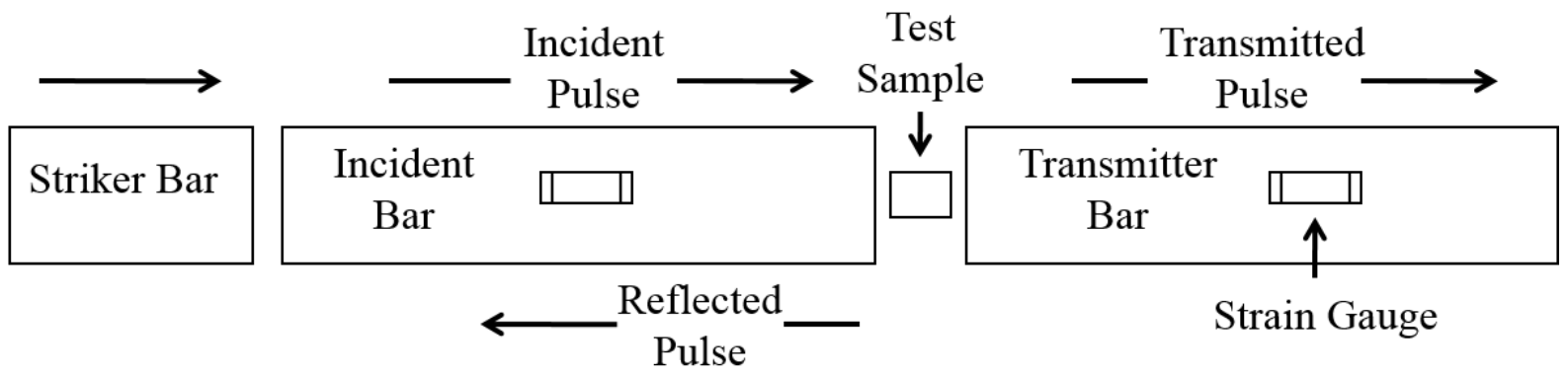

Figure 2. Schematic Split-Hopkinson test set up.

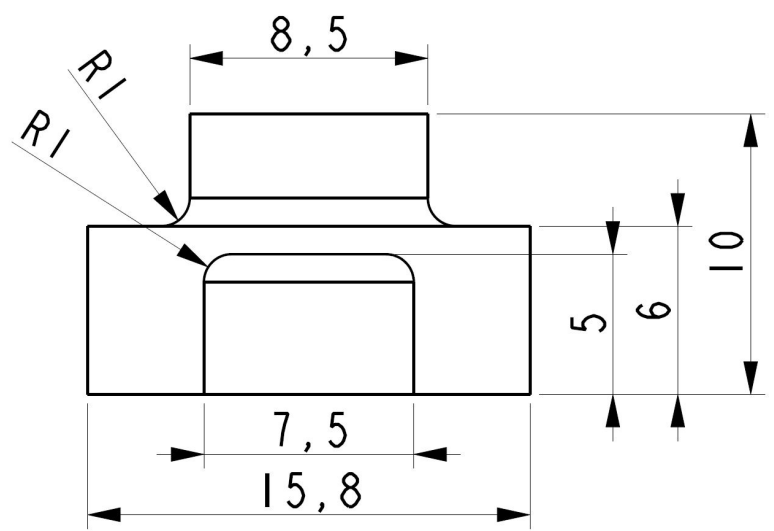

Figure 3. Sketch of the top-hat sample with $1 \mathrm{~mm}$ radius in the geometry transition. 


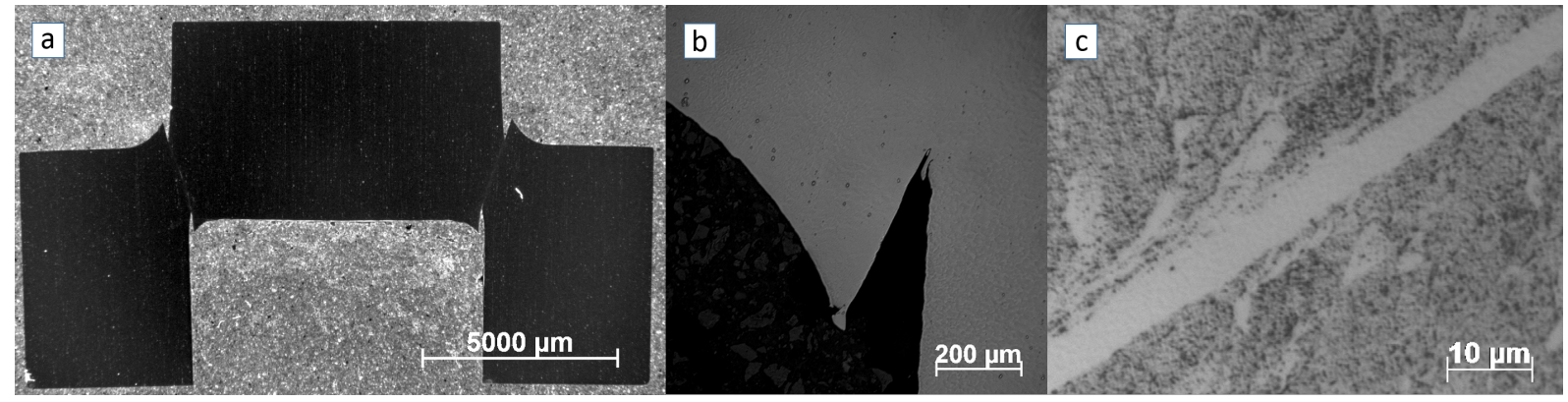

Figure 4. (a) Cross section of Top-Hat and close ups of (b) shear initiated displacement and (c) shear band

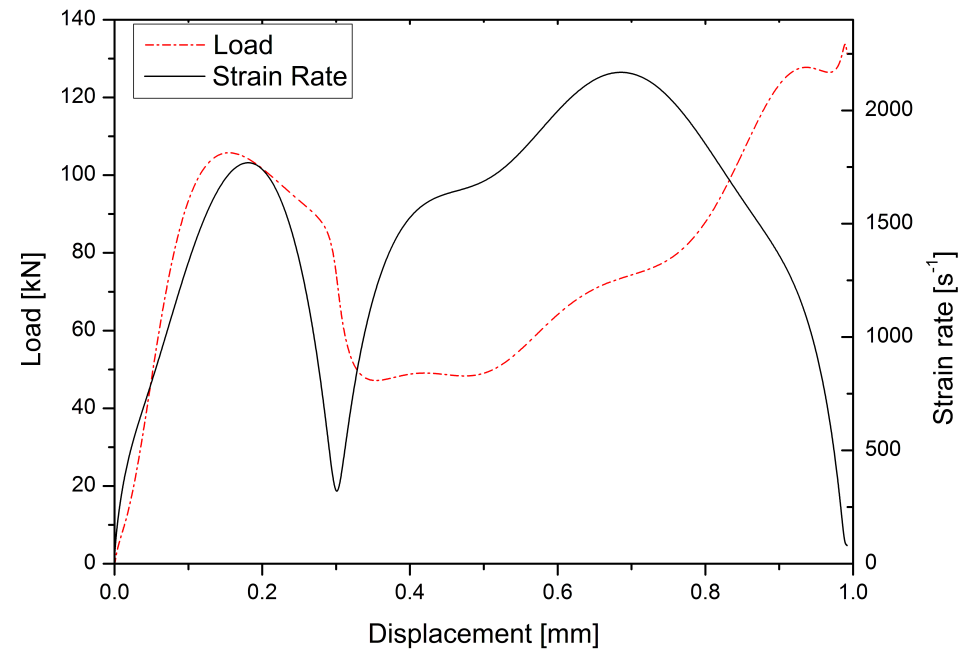

Figure 5. Mechanical response of the Top-Hat testing, Global response showing load and strain rate during the compression. 


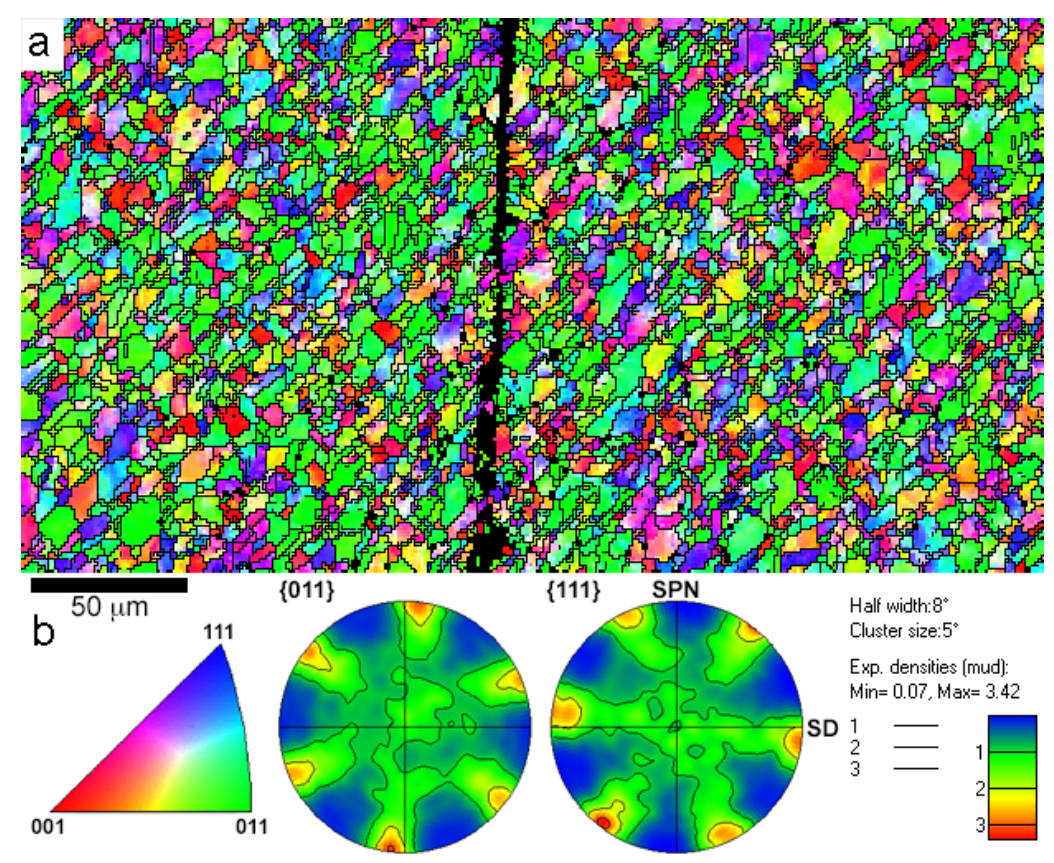

Figure 6. (a) EBSD map of the SAZ, including the shear band in the centre, and the corresponding pole figures (b), indicating a strong shear texture.

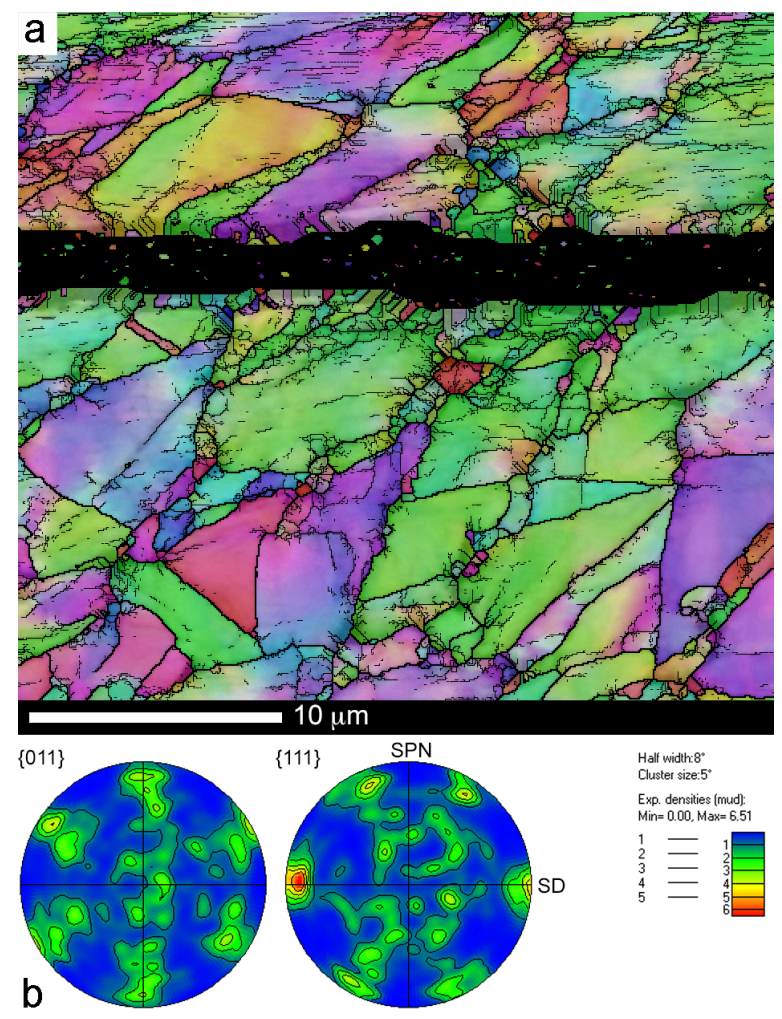

Figure 7. (a) EBSD map (superimposed on the band contrast) of the region adjacent to the shear band. Thin and thick black lines are low-angle and high angle grain boundaries, respectively. The absence of a distinct transition zone can be noted, and the pole figures (b) show a maintained shear texture. Colour legend as in Fig. 6. 


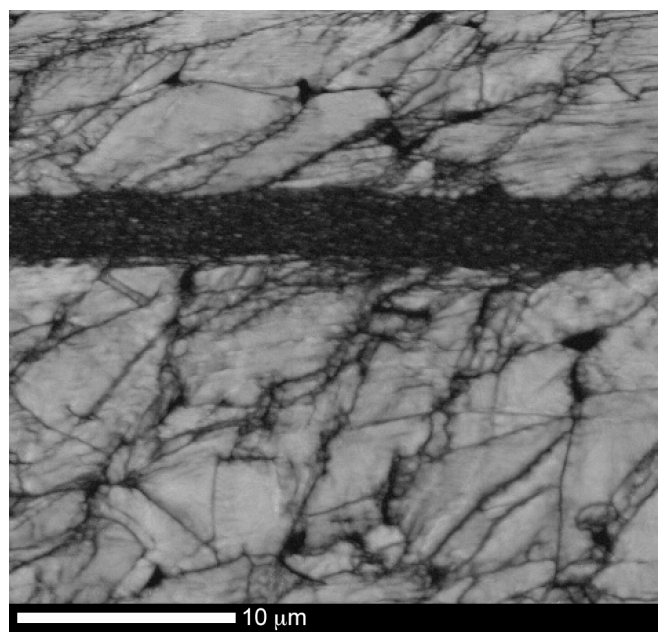

Figure 8. Band contrast map corresponding to Fig. 7(a).
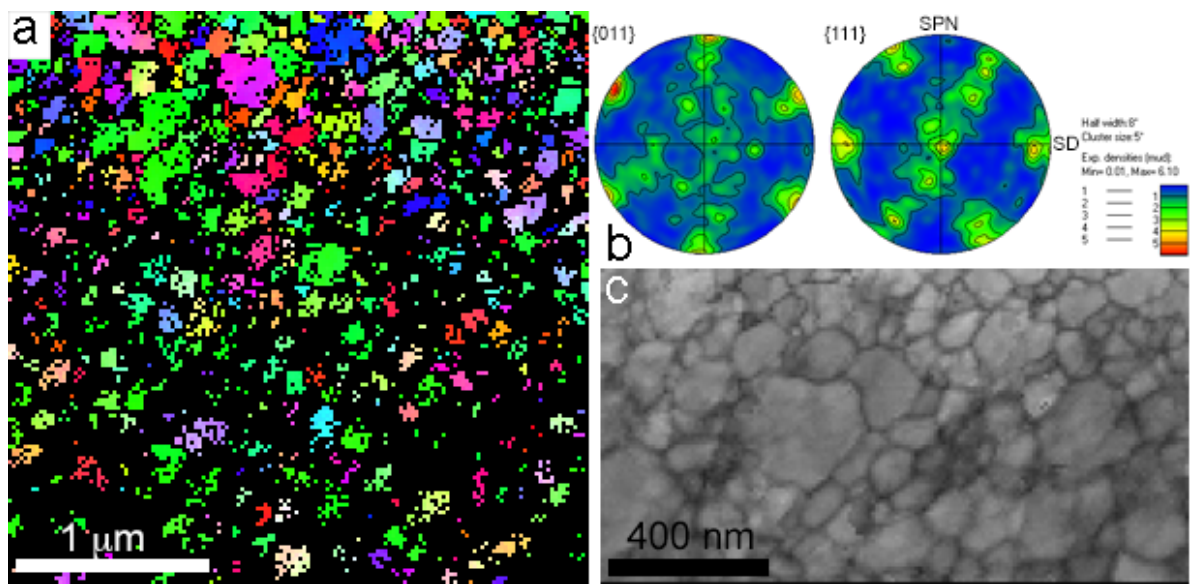

Figure 9. (a) TKD map and band contrast (c) map from within the shear band, and the corresponding pole figures (b) indicating a shear textures. The band contrast map shows the presence of equiaxed ultra-fine grains (100-300 nm). Colour legend as in Fig. 6.
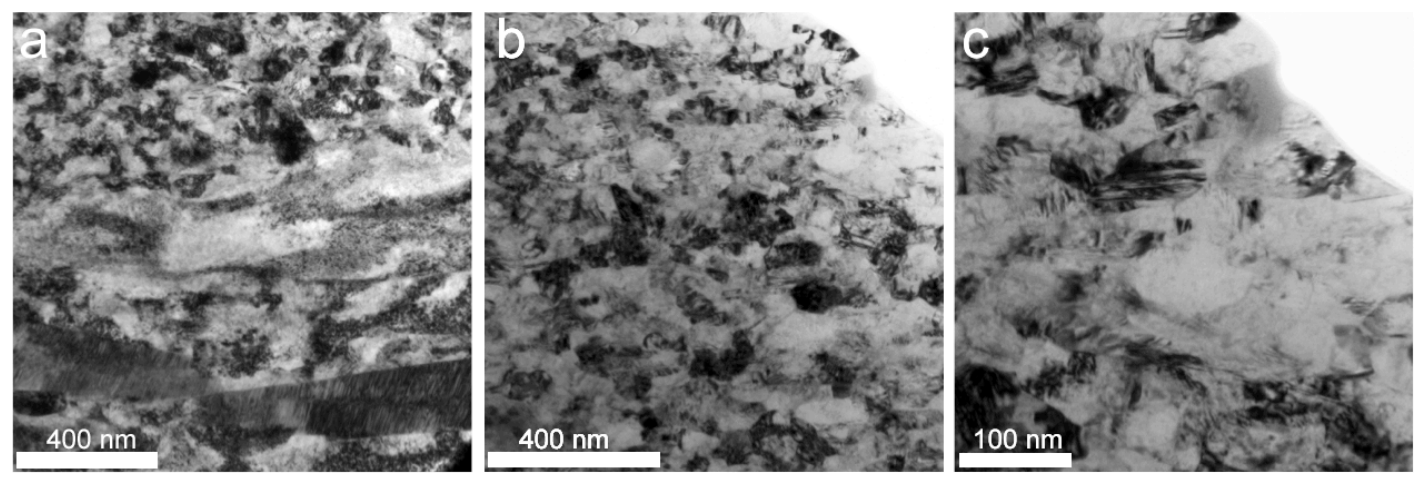

Figure 10. TEM bright-field micrographs: (a) Transition between the band and surrounding material in the top-hat specimen. (b) Overview of the ultra-fine grained structure in the band. (c) Higher magnification showing grains as small as $20 \mathrm{~nm}$. 


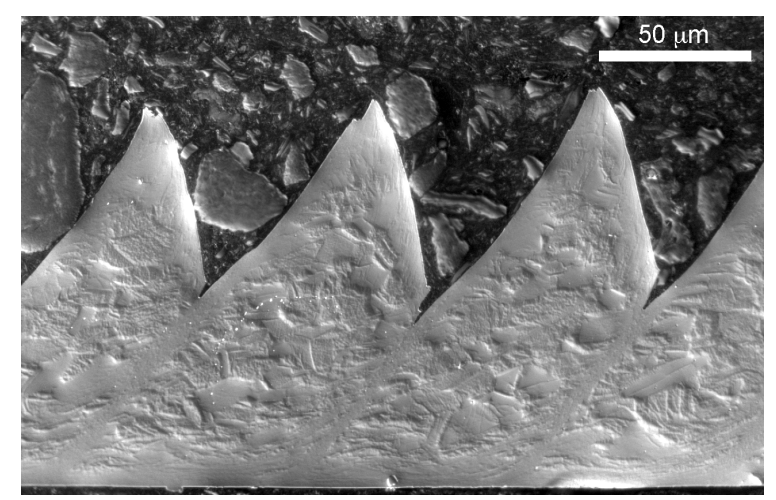

Figure 11. Metal cutting chip, cut at $240 \mathrm{~m} / \mathrm{min}$ and $0.1 \mathrm{~mm} / \mathrm{rev}$ showing segments with shear band in between.
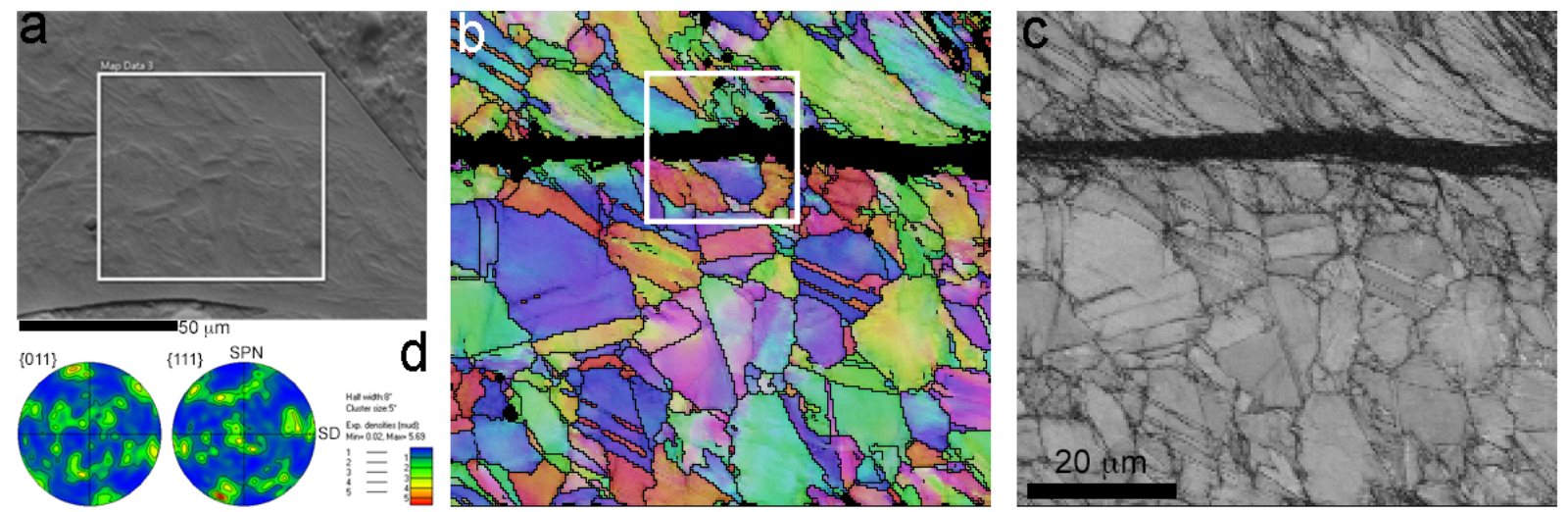

Figure 12. EBSD (b) and band contrast (b) maps of the region indicated in the box in (a). The black lines are HAGBs. As for the top-hat specimens, a shear texture is observed (d), and no clear transition zone can be seen. IPF colour legend as in Fig. 6.
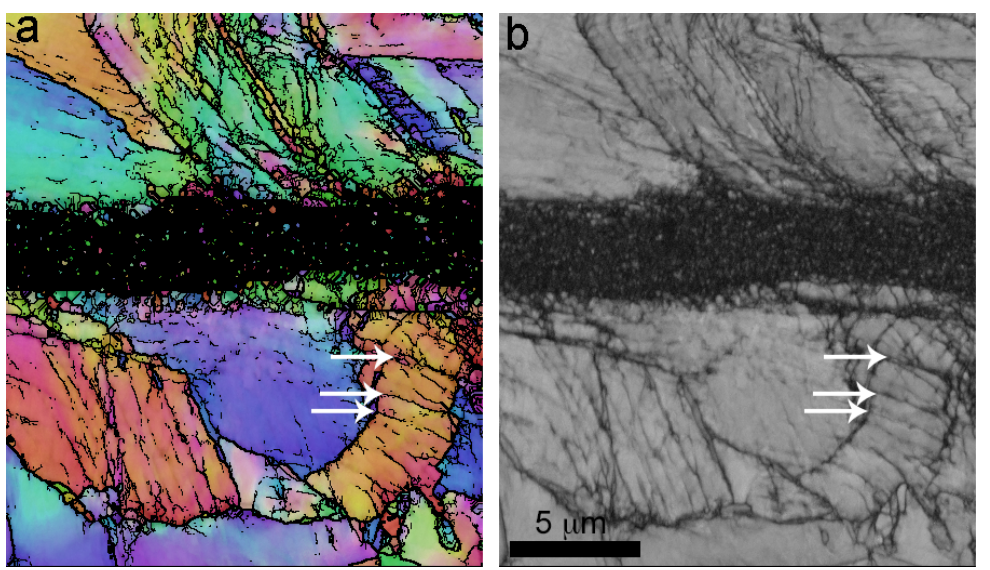

Figure 13. (a) Higher magnification EBSD map close to the shear band in the chip (boxed region in Fig. 12). Thin and thick black lines are low-angle and high angle grain boundaries, respectively. The band contrast map (b) shows a very narrow transition zone. Arrows indicate the occurrence of grain subdivision through shearing. Colour legend as in Fig. 6. 


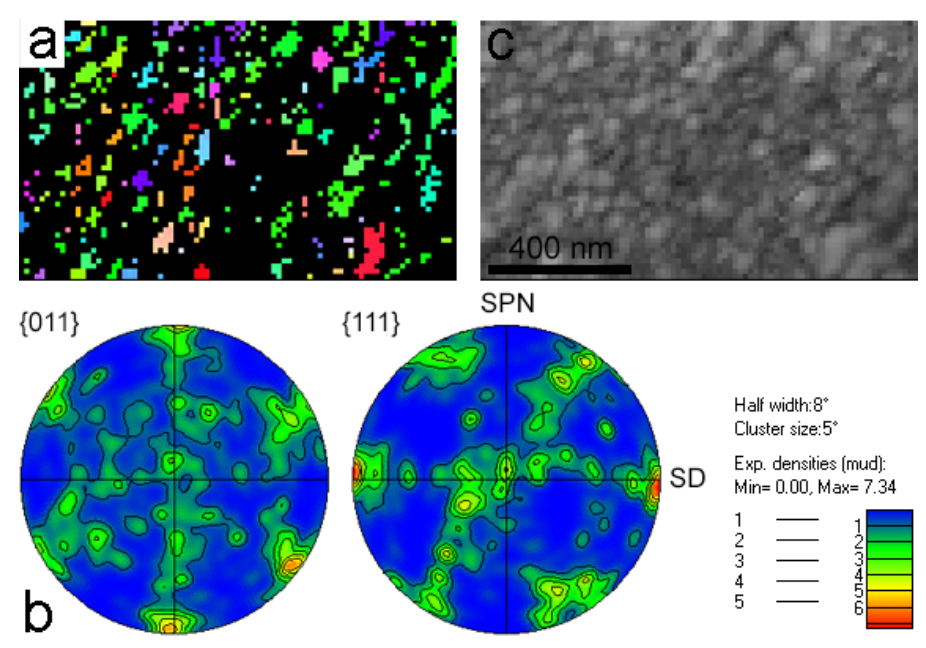

Figure 14. (a) TKD map from within the band in the chip, and the corresponding pole figures (b) showing a shear texture. The band contrast (c) shows ultra-fine equiaxed grains. Colour legend as in Fig. 6.
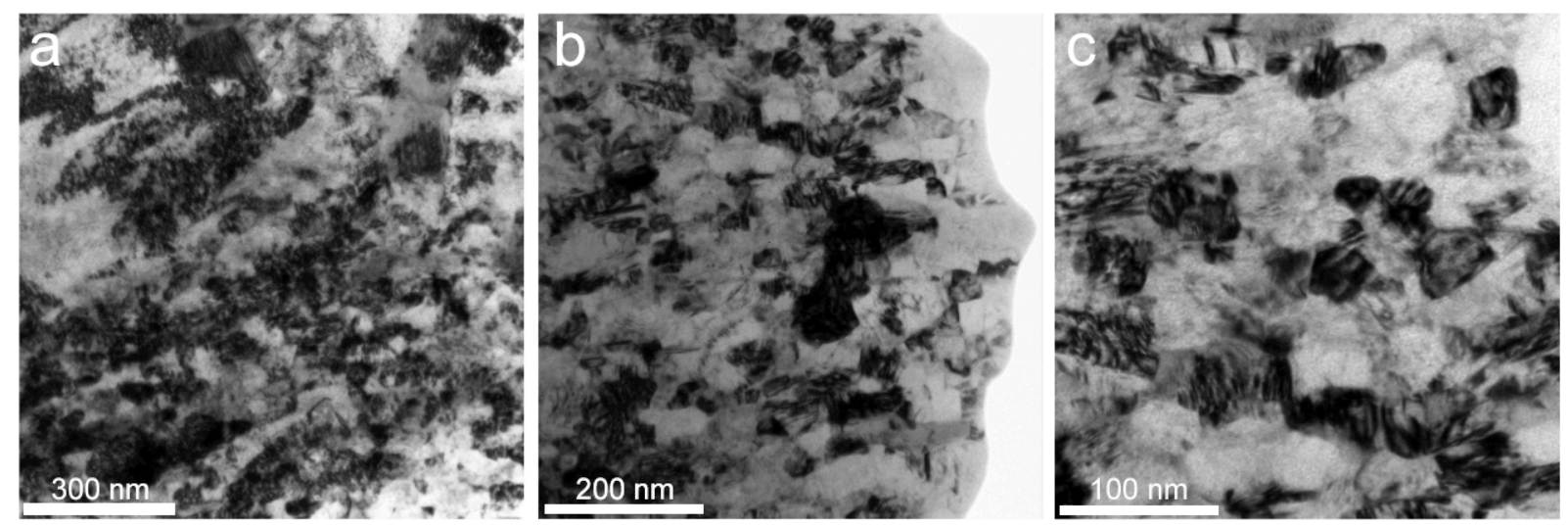

Figure 15. TEM bright-field micrographs: (a) Transition between the band and surrounding material in the machining chip. (b) Overview of the ultra-fine grained structure in the band. (c) Higher magnification showing grains as small as $20 \mathrm{~nm}$. 